\title{
Quantum criticality of magnetic catalysis in two-dimensional correlated Dirac fermions
}

\author{
Yasuhiro Tada $\oplus^{*}$ \\ Institute for Solid State Physics, University of Tokyo, Kashiwa 277-8581, Japan
}

(Received 29 April 2020; revised 5 July 2020; accepted 13 August 2020; published 3 September 2020)

\begin{abstract}
We study quantum criticality of the magnetic-field-induced charge density wave (CDW) order in correlated spinless Dirac fermions on the $\pi$-flux square lattice at zero temperature as a prototypical example of the magnetic catalysis, by using the infinite density matrix renormalization group. It is found that the CDW order parameter $M(B)$ exhibits an anomalous magnetic field $(B)$ scaling behavior characteristic of the $(2+1)$-dimensional chiral Ising universality class near the quantum critical point, which leads to a strong enhancement of $M(B)$ compared with a mean-field result. We also establish a global phase diagram in the interaction-magnetic field plane for the fermionic quantum criticality.
\end{abstract}

DOI: 10.1103/PhysRevResearch.2.033363

\section{INTRODUCTION}

Correlated Dirac semimetals are one of the most fundamental systems not only in condensed matter physics but also in high-energy physics. They exhibit semimetal-insulator transitions at some critical strength of interactions $V=V_{c}>$ 0 at zero temperature, and magnetic/charge ordered states are stabilized for stronger interactions $V>V_{c}$ [1-22]. These ordered phases correspond to the dynamically massive states with broken chiral symmetry in high-energy physics. Interestingly, criticality of the quantum phase transitions are qualitatively different from those of conventional magnetic/charge orders in purely bosonic systems, which is dubbed fermionic criticality. In these criticalities, bosonic order parameter fluctuations are intimately coupled with gapless Dirac fermions, which results in nontrivial quantum critical behaviors depending on fermionic degrees of freedom in addition to the order parameter symmetry and dimensionality of the system [23]. The fermionic criticality has been discussed extensively by various theoretical methods such as lattice model simulations [1-14] and renormalization group calculations [15-21], and now the basic understanding of these systems has been well established.

Correlation effects in a Dirac system become even more significant in presence of an applied magnetic field. It is known that an infinitesimally small magnetic field induces a magnetic/charge order for any nonzero interaction $V$, which is called the "magnetic catalysis" [24-44]. A uniform magnetic field $B$ will effectively reduce spatial dimensionality $d$ of the system via the Landau quantization, $d \rightarrow d-2$. Therefore, the system becomes susceptible to formation of a bound

\footnotetext{
*tada@issp.u-tokyo.ac.jp

Published by the American Physical Society under the terms of the Creative Commons Attribution 4.0 International license. Further distribution of this work must maintain attribution to the author(s) and the published article's title, journal citation, and DOI.
}

state by interactions. For example, in the $(2+1)$-dimensional Gross-Neveu-Yukawa-type models, it is shown that in the limit of the large number of fermion flavors $N_{f}$ corresponding to a mean-field approximation, the order parameter behaves as $M(B) \sim B$ for weak interactions $V \ll V_{c}, M(B) \sim \sqrt{B}$ near the critical point $V=V_{c}$, and $M(B)-M(0) \sim B^{2}$ for strong interactions $V \gg V_{c}$. Although the magnetic catalysis was first studied in high-energy physics, it was also discussed in condensed matter physics, especially for graphene and related materials [36-44]. Recently, there have emerged a variety of candidate Dirac materials with strong electron correlations [45-49] which could provide a platform for an experimental realization of the magnetic catalysis, and a detailed understanding of this phenomenon is an important issue.

However, most of the previous theoretical studies for systems near quantum criticality are based on perturbative approximations [24-44,50], and the true critical behaviors beyond the large $N_{f}$ limit are rather poorly explored. This is in stark contrast to the correlated Dirac systems without a magnetic field, for which there are extensive numerical simulations in addition to the field theoretical calculations, and critical behaviors have been well established [1-22]. Therefore, further theoretical developments are required for clarifying the genuine nature of the quantum critical magnetic catalysis.

In this work, we study quantum criticality of the fieldinduced charge density wave (CDW) order in spinless Dirac fermions on the two-dimensional $\pi$-flux square lattice, which is one of the simplest realizations of the magnetic catalysis. We use a nonperturbative numerical technique, infinite density matrix renormalization group (iDMRG) which can directly describe spontaneous $\mathbb{Z}_{2}$ symmetry breaking of the CDW order [51-56]. It is found that the order parameter exhibits an anomalous critical behavior, which characterizes the fermionic criticality as clarified by a scaling argument with respect to the magnetic length. Based on these observations, we establish a global phase diagram for the ground state near the quantum critical point. 


\section{MODEL}

We consider spinless fermions on a $\pi$-flux square lattice at half-filling under a uniform magnetic field. There are two Dirac cones in the Brillouin zone, and each Dirac fermion has two (sublattice) components, which corresponds to a case where the total number of Dirac fermion components is four, similarly to the honeycomb lattice model [3-8]. The Hamiltonian is given by

$$
H=-\sum_{\langle i, j\rangle} t_{i j} c_{i}^{\dagger} c_{j}+V \sum_{\langle i, j\rangle} n_{i} n_{j},
$$

where $\langle i, j\rangle$ is a pair of the nearest-neighbor sites and the energy unit is $t=1$. The hopping is $t_{i j}=t e^{i \pi y_{i}} \exp \left(i A_{i j}\right)$ along the $x$ direction on the $y=y_{i}$ bond and $t_{i j}=t \exp \left(i A_{i j}\right)$ along the $y$ direction. The vector potential is given in the string gauge [57] with the period $L_{x}^{\prime} \times L_{y}$ where $L_{x}^{\prime}$ is the superlattice unit period used in the iDMRG calculations for the system size $L_{x} \times L_{y}=\infty \times L_{y}$. Typically, we use $L_{x}^{\prime}=20$ for $L_{y}=6$ and $L_{x}^{\prime}=10$ for $L_{y}=10$ (see also Appendix A). $A_{i j}=0$ corresponds to the conventional $\pi$-flux square lattice without an applied field, while $A_{i j} \neq 0$ describes an applied magnetic field for a plaquette $p, B_{p}=\sum_{\langle i j\rangle \in p} A_{i j}$. The magnetic field is spatially uniform and an integer multiple of a unit value $B=n \times \delta B \quad\left(n=1,2, \ldots, L_{x}^{\prime} L_{y}\right)$ allowed by the superlattice size, where $\delta B=2 \pi / L_{x}^{\prime} L_{y}$. The lattice constant $a$ as a length unit and the electric charge $e$ have been set as $a=1, e=1$, and the magnetic field is measured in the unit of $B_{0}=2 \pi$.

The $V$ term is a repulsive nearest-neighbor interaction leading to the CDW state, and the quantum phase transition with $B=0$ takes place at $V=V_{c} \simeq 1.30 t$ according to the quantum Monte Carlo calculations for the bulk twodimensional system, where the criticality belongs to the $(2+1)$-dimensional chiral Ising universality class [3-8]. On the other hand, our cylinder system is anisotropic, and the CDW quantum phase transition at $B=0$ is simply a $(1+1)$ dimensional Ising transition and critical interaction strength depends on the system size $L_{y}$, which may be regarded as a finite-size effect [58]. However, the system can be essentially two-dimensional in space under a magnetic field when the magnetic length $l_{B}=1 / \sqrt{B}$ becomes shorter than the system size $L_{y}$. We will use this property to discuss the $(2+$ 1)-dimensional criticality. Note that the critical interaction strength $V_{c} \simeq 1.30 t$ will be confirmed later within the present framework.

In the following, we focus on the CDW order parameter,

$$
M=\left|\frac{1}{L_{x}^{\prime} L_{y}} \sum_{i}(-1)^{|i|} n_{i}\right|,
$$

where the summation runs over the superlattice unit cell. In the iDMRG calculation, we introduce a finite bond dimension $\chi$ up to $\chi=1600$ as a cutoff to approximate the ground-state wave function in the form of a matrix product state, and we can obtain the true ground state by a careful extrapolation to $\chi \rightarrow \infty$ from the finite $\chi$ results [51-56] (see also Appendix A). Generally, iDMRG with finite $\chi$ gives a good approximation especially when the system considered is gapful. As we will show, an extrapolation to $\chi \rightarrow \infty$ works

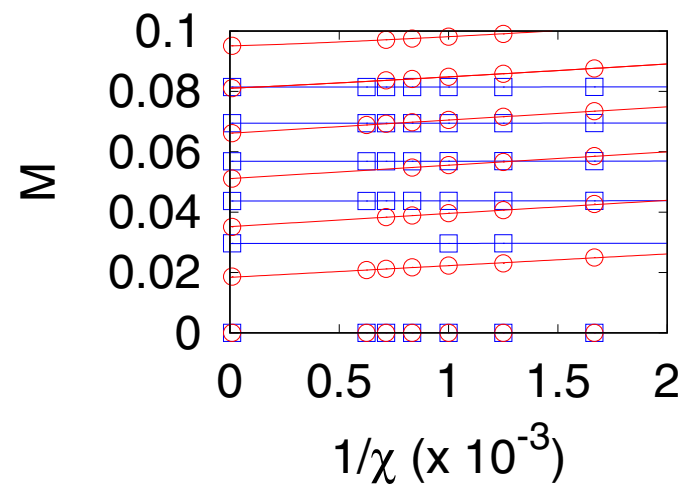

FIG. 1. Extrapolation of the CDW order parameter $M(B)$ for the $\chi \rightarrow \infty$ limit at $V=0.50 t$. The blue (red) symbols are for $L_{y}=6\left(L_{y}=10\right)$, and the curves are power-law fittings. Each curve corresponds to a magnetic field in the range $0 \leqslant B \leqslant 0.06 B_{0}$.

well, because our system has a gap in presence of a nonzero $B$ due to the magnetic catalysis of the broken discrete symmetry $\mathbb{Z}_{2}$ where there is no gapless Nambu-Goldstone mode. For a comparison, we also discuss a two-leg ladder system with $L_{y}=2$ in Appendix B.

\section{AWAY FROM QUANTUM CRITICAL POINT}

Before discussing quantum criticality, we investigate the magnetic catalysis when the system is away from the critical point. First, we consider a weak interaction $V=0.50 t<V_{c}=$ $1.30 t$ for which the system at $B=0$ is a Dirac semimetal renormalized by the interaction. As exemplified in Fig. 1, dependence of $M(B)$ on the bond dimension $\chi$ used in the calculation is negligibly small for $L_{y}=6$, and it can be safely extrapolated to $\chi \rightarrow \infty$ even for $L_{y}=10$. Standard deviations of the extrapolations are less than $1 \%$ and within the symbols. Such an extrapolation can be done also for other values of $V$ as mentioned before, and all results shown in this study are extrapolated ones.

In Fig. 2(a) we show the CDW order parameter $M$ extrapolated to $\chi \rightarrow \infty$ for the system sizes $L_{y}=6$ and $L_{y}=10$ at $V=0.50 t$. The calculated results almost converge for $L_{y}=$ 6,10 and are independent of the system size, except for $B=0$ where there is a finite-size effect due to $l_{B}=\infty$, although there is some accidental deviation around $B \simeq 0.1 B_{0}$. Therefore, these results give the CDW order parameter essentially in the thermodynamic limit $L_{y} \rightarrow \infty$. In order to understand impacts of quantum fluctuations, we also performed a meanfield calculation for a comparison [59]. The critical interaction within the mean-field approximation is found to be $V_{c}=0.78 t$, and the interaction $V=0.30 t$ corresponds to the same coupling strength in terms of the normalized interaction $g=\left(V-V_{c}\right) / V_{c}=-0.62$. The iDMRG reuslts of $M$ (blue symbols) are larger than the corresponding mean-field results (red symbols), $M>M_{\mathrm{MF}}$, which suggests that quantum fluctuations enhance the order parameter even for the present weak $V$. It is noted that the order parameter behaves roughly as $M(B) \sim B$ as seen for small $B$, which is consistent with the large $N_{f}$ field theory [24-31]. 

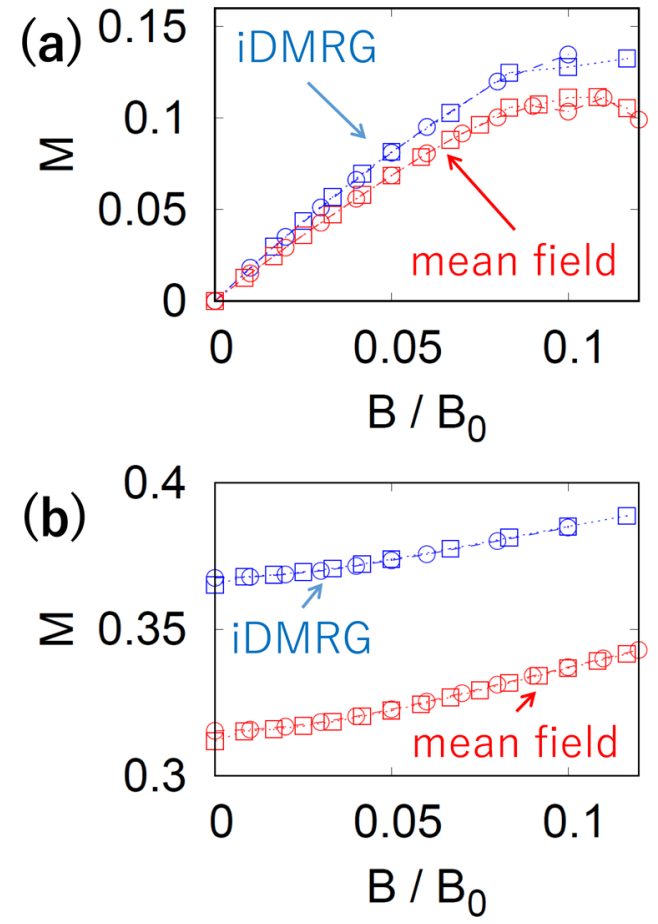

FIG. 2. (a) The CDW order parameter $M$ at a weak coupling. The blue symbols are the iDMRG results at $V=0.50 t<V_{c}$ for $L_{y}=6$ (squares) and $L_{y}=10$ (circles), and the red symbols are the meanfield results $(V=0.30 t)$ for the same system sizes. (b) $M$ at a strong coupling $V=2.0 t>V_{c}$ calculated by iDMRG (blue) and $V=1.20 t$ by the mean-field approximation (red). The interactions for iDMRG and the mean-field approximation correspond to the same value of the normalized coupling constant $g$.

Similarly to the weak interaction case, the CDW order parameter $M$ calculated by iDMRG (blue symbols) is enhanced at a strong interaction $V=2.0 t>V_{c}$ compared to the mean-field result $M_{\mathrm{MF}}$ (red symbols) at the corresponding interaction $V=1.20 t$ (or equivalently $g=0.54$ ) as shown in Fig. 2(b). However, this is governed by the $B=0$ values, and an increase of $M(B)$ by the magnetic field is roughly comparable to that of $M_{\mathrm{MF}}(B)$. The result that $M>M_{\mathrm{MF}}$ already at $B=0$ is because they behave as $M(V, B=0) \sim g^{\beta}$ with $\beta \simeq 0.5 \sim 0.6<1[3-8]$ while $M_{\mathrm{MF}}(V, B=0) \sim g^{\beta_{\mathrm{MF}}}$ with $\beta_{\mathrm{MF}}=1$ near the quantum critical point, and these critical behaviors essentially determine magnitudes of the CDW order parameters away from the critical points. For $B \neq 0$, the order parameter behaves roughly as $M(B)-M(0) \sim B^{2}$ in agreement with the large $N_{f}$ field theory [24-31].

\section{NEAR QUANTUM CRITICAL POINT}

In this section, we discuss quantum criticality of the magnetic catalysis based on a variant of finite-size scaling ansatzes. Then, we establish a global phase diagram around the quantum critical point in the interaction-magnetic field plane, in close analogy with the well-known finite temperature phase diagram near a quantum critical point.

\section{A. Scaling argument}

The enhancement of $M(B)$ by the quantum fluctuations can be even more pronounced near the quantum critical
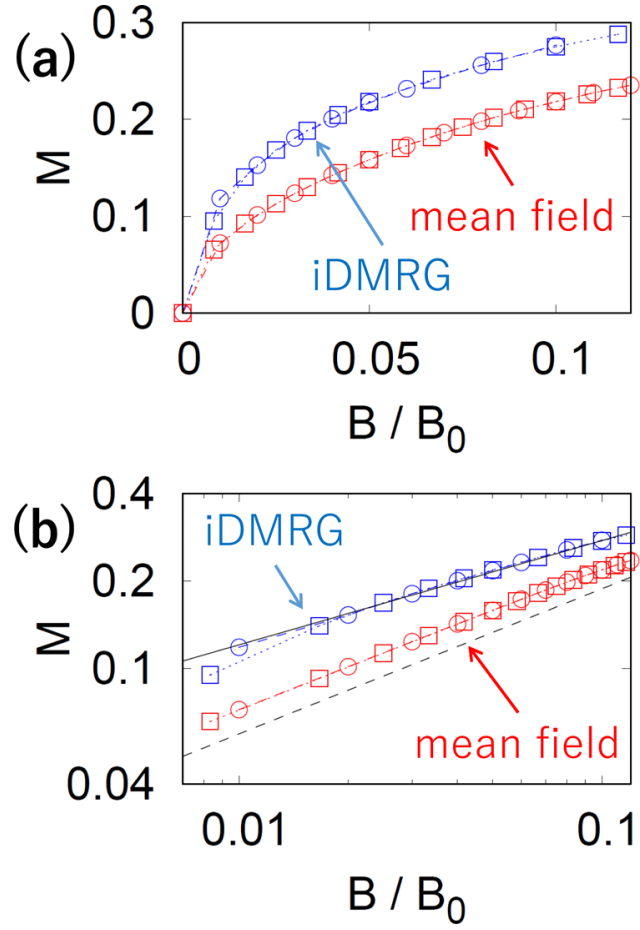

FIG. 3. (a) The CDW order parameter $M$ at the quantum critical point $V=V_{c}=1.30 t$ calculated by iDMRG together with the meanfield result at $V=0.78 t$ corresponding to $g=0$. Definitions of the symbols are the same as in Fig. 2. (b) $M$ in the log-log plot. The black solid line is the power law fitting $M \sim B^{0.355}$, and the black dashed line is the large $N_{f}$ result $M \sim \sqrt{B}$ shown for the eyes.

point. Figure 3 shows the CDW order parameter at $V=V_{c}=$ $1.30 t$ (blue symbols) together with the mean-field result for $V=0.78 t$ (red symbols), corresponding to $g=0$. Clearly, the iDMRG result is significantly larger than the mean-field result, and the enhancement is much stronger than that in the weak interaction case. There are some deviations between the results for $L_{y}=6$ and $L_{y}=10$ for small magnetic fields, $B \lesssim 0.01 B_{0}$, due to a long magnetic length $l_{B}$, and the CDW order gets more strongly stabilized when the system size $L_{y}$ increases from $L_{y}=6$ to $L_{y}=10$. This should be a general tendency since the CDW phase at $B=0$ extends to a smaller interaction region when the system size increases [58]. From this observation, we can discuss scaling behaviors of the CDW order parameter in the thermodynamic limit as a function of $B$ near the quantum critical point. Indeed, as shown in Fig. 3(b), the calculated $M$ except for the smallest values of $B$ converge for different system sizes $L_{y}=6,10$, and $M(B)$ exhibits a power-law behavior for $0.02 B_{0} \lesssim B \lesssim 0.1 B_{0}$. The finite-size effects are negligible in this range of the magnetic field, and furthermore the scaling behavior would hold for smaller magnetic fields down to $B=0$ in a thermodynamic system $L_{y} \rightarrow \infty$, since $M\left(L_{y}=10\right)$ shows the scaling behavior in a wider region of $B$ than $M\left(L_{y}=6\right)$ does. If we focus on $0.02 B_{0} \lesssim B \lesssim 0.1 B_{0}$ in Fig. 3, we obtain the anomalous scaling behavior $M(B) \sim B^{0.355(6)}$ by power-law fittings for different sets of data points. This is qualitatively different from the mean-field (or equivalently large $N_{f}$ limit) result 
$M_{\mathrm{MF}} \sim \sqrt{B}$, which eventually leads to the strong enhancement of $M(B)$ compared to $M_{\mathrm{MF}}(B)$.

The calculated magnetic field dependence of the CDW order parameter near $V=V_{c}$ implies a scaling relation characteristic of the quantum criticality. Here we propose a scaling ansatz for the leading singular part of the ground-state energy density of a thermodynamically large $(2+1)$-dimensional system,

$$
\varepsilon_{\text {sing }}\left(g, h, l_{B}^{-1}\right)=b^{-D} \varepsilon_{\text {sing }}\left(b^{y_{g}} g, b^{y_{h}} h, b l_{B}^{-1}\right),
$$

where $D=2+z=2+1=3$ with $z=1$ being the dynamical critical exponent and $h$ is the conjugate field to the CDW order parameter $M$. The exponents $y_{g, h}$ are corresponding scaling dimensions, and the scaling dimension of the magnetic length is assumed to be one, as will be confirmed later. For a thermodynamic system, the magnetic length $l_{B}$ will play a role of a characteristic length scale similarly to a finite system size $L$. Then a standard argument similar to that for a finite-size system at $B=0$ applies, leading to

$$
M\left(g=0, l_{B}^{-1}\right) \sim\left(l_{B}^{-1}\right)^{\beta / v} \sim B^{\beta / 2 v},
$$

where $\beta$ and $v$ are the critical exponents at $B=0$ for the order parameter $M\left(g, l_{B}^{-1}=0\right) \sim g^{\beta}$ and the correlation length $\xi\left(g, l_{B}^{-1}=0\right) \sim g^{-v}$. One sees that this coincides with the familiar finite-size scaling if we replace $l_{B}$ with a system size $L$ [60]. The critical exponents of the CDW quantum phase transition in $(2+1)$ dimensions are $\beta=v=1$ in the meanfield approximation, and the resulting $M \sim B^{0.5}$ is consistent with our mean-field numerical calculations [61]. The true critical exponents for the present $(2+1)$-dimensional chiral Ising universality class with four Dirac fermion components have been obtained by the quantum Monte Carlo simulations at $B=0$ and are given by $(\beta=0.53, v=0.80)[3,4]$, which was further supported by the infinite projected entangled pair state calculation [14]. Other quantum Monte Carlo studies with different schemes and system sizes give $(\beta=0.63, v=0.78)[5,6],(\beta=0.47, v=0.74)[7]$, and $(\beta=0.67, v=0.88)$ [8]. These exponents lead to $\beta / 2 v=$ $0.33,0.40,0.32,0.38$ respectively, and the scaling behavior of $M(B)$ found in our study falls into this range and is consistent with them.

The homogeneity relation (3) and the critical exponent can be further confirmed by performing a data collapse. According to Eq. (3), the CDW order parameter for general $g$ is expected to behave as

$$
M\left(g, l_{B}^{-1}\right)=l_{B}^{-\beta / v} \Phi\left(g l_{B}^{1 / v}\right),
$$

where $\Phi(\cdot)$ is a scaling function. This is a variant of the finite-size scaling similarly to Eq. (4). When performing a data collapse, we use the results for $0.02 B_{0} \lesssim B \lesssim 0.1 B_{0}$ so that finite-size effects are negligible. As shown in Fig. 4, the calculated data well collapse into a single curve, and the critical exponents are evaluated as $\beta=0.54(3), v=0.80(2)$ with $V_{c}=1.30(2) t$. This gives $\beta / 2 v=0.34(2)$, which is consistent with $\beta / 2 v=0.36$ obtained from $M\left(V=V_{c}, B\right)$ at the quantum critical point (Fig. 3). Our critical exponents are compatible with those obtained previously by the numerical calculations as mentioned above and roughly with those by the field theoretic calculations [1-22]. Our numerical calculations

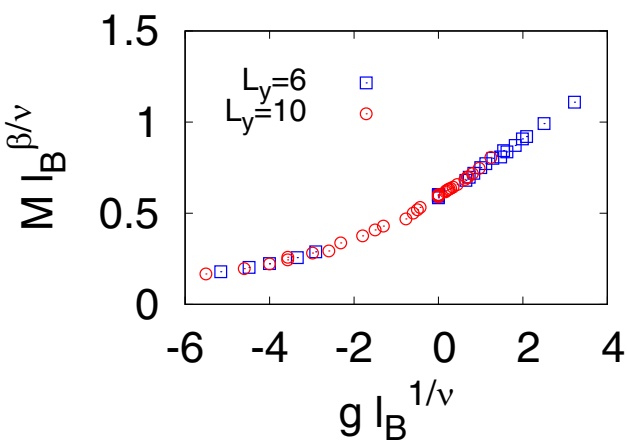

FIG. 4. Scaling plot of the CDW order parameter $M(V, B)$ in terms of $g=\left(V-V_{c}\right) / V_{c}$ and $l_{B}=1 / \sqrt{B}$. The blue squares are for $L_{y}=6$ and red circles for $L_{y}=10$.

for the $(2+1)$-dimensional criticality are limited to rather small magnetic lengths $l_{B}$ bounded by the system size $L_{y}$, and we expect that more accurate evaluations of the critical exponents would be possible for larger $L_{y}$ with controlled extrapolations to $\chi \rightarrow \infty$.

The successful evaluation of the critical exponents strongly verifies the scaling ansatz (3). Although the scaling ansatz may be intuitively clear and similar relations were discussed for the bosonic Ginzburg-Landau-Wilson theory in the context of the cuprate high- $T_{c}$ superconductivity [62-64], its validity is a priori nontrivial, and there have been no nonperturbative analyses even for the well-known bosonic criticality. This is in stark contrast to the conventional finite system size scaling at $B=0$, which has been well established for various systems [60]. The present study is a first nonperturbative analysis of the $l_{B}$-scaling relation, providing a clear insight from a statistical physics point of view for the quantum critical magnetic catalysis. Besides, the scaling ansatz could be used as a theoretical tool for investigating some critical phenomena similarly to the recently developed finite correlation length scaling in tensor network states (see also Appendix B) $[14,58,65,66]$. Based on this observation, one could evaluate critical behaviors of the magnetic catalysis in other universality classes in $(2+1)$ dimensions, such as $\mathrm{SU}(2)$ and $\mathrm{U}(1)$ symmetry breaking with a general number of Dirac fermion components, by using the critical exponents obtained for the phase transitions at $B=0$ [1-22]. It would be a future problem to clarify the exact condition for the $l_{B}$ scaling to hold in general cases.

\section{B. Phase diagram}

The above discussions can be summarized into a global phase diagram near the quantum critical point in the $V$ - $B$ plane at zero temperature as shown in Fig. 5. Here we mainly focus on the critical behaviors of the order parameter but not on phase boundaries. In this phase diagram, there are two length scales: one is the correlation length of the CDW order parameter $\xi$ at $B=0$, and the other is the magnetic length $l_{B}$. One can compare it with the familiar finite temperature phase diagram near a quantum critical point [67-69]. The length scale $l_{B}$ in our case corresponds to a system size along the imaginary time, $L_{\tau}=1 / T$, in a standard quantum critical system at finite temperature $T$. In a finite temperature system, anomalous 


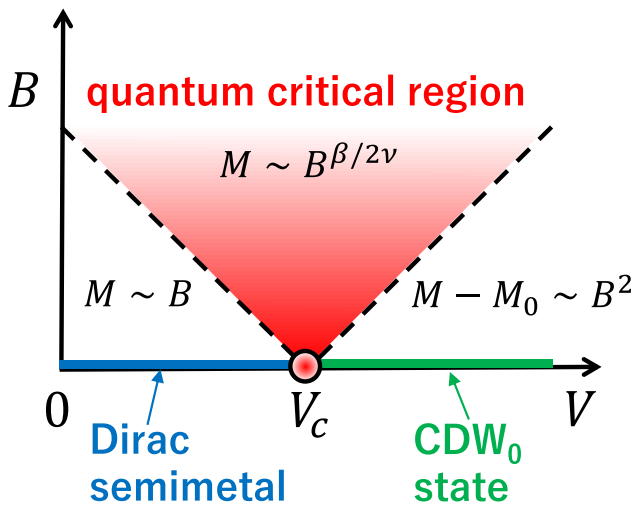

FIG. 5. Schematic phase diagram in the $V-B$ plane at zero temperature and the $B$ dependence of $M(V, B)$ for fixed $V$ in each region. The CDW state at $B=0$ is denoted as $\mathrm{CDW}_{0}$ and $M_{0}(V)=$ $M(V, B=0) \sim\left(V-V_{c}\right)^{\beta}$. The crossover boundaries (dashed lines) are characterized by $l_{B} \simeq \xi$.

finite temperature behaviors are seen when the dynamical correlation length $\xi_{\tau} \sim \xi^{z}$ becomes longer than the temporal system size, $\xi_{\tau} \gg L_{\tau}$, so that the critical singularity is cut off by $L_{\tau}$ in the imaginary time direction [67-69]. Similarly in the present system at $T=0$, physical quantities will exhibit anomalous $B$ dependence when the spatial correlation length $\xi$ is longer than the magnetic length, $\xi \gg l_{B}$, and the critical singularity is cut off by $l_{B}$ in the spatial direction. In this way, we can understand the scaling behavior $M \sim l_{B}^{-\beta / \nu} \sim$ $B^{\beta / 2 v}$ in close analogy with the finite temperature scaling behaviors associated with a quantum critical point at $B=0$. On the other hand, the order parameter shows conventional $B$ dependence, $M(B) \sim B$ or $M(B)-M(0) \sim B^{2}$, when the system is away from the quantum critical point, $\xi \ll l_{B}$. We note that our phase diagram would be qualitatively applicable to an interacting Dirac system with a general flavor number $N_{f}$ including $N_{f} \rightarrow \infty$ with $\beta=v=1$ [24,25]. It is also noted that the Dirac semimetal phase will be extended to a $B \neq 0$ region at finite low temperature [24,25,32-35,42-44], and the critical behaviors can be modified as will be briefly discussed later.

We would also expect that a similar phase diagram could be seen even in a system with long-range interactions such as QED-like theories in the massless limit, because it is considered that criticality of a quantum phase transition in a $(2+1)$ dimensional Dirac system driven by a short-range interaction is not affected by the long-range Coulomb interaction $[9,19]$. It is noted that, while the Coulomb interaction is (marginally) irrelevant at the transition point, it will play an important role at a weak coupling regime, and an order parameter could behave as $M \sim \sqrt{B}$ even for any small coupling [24,25].

\section{Discussion}

In this section, we discuss several issues in the magnetic catalysis including possible future studies.

\section{Comparison with conventional finite-size effects}

In the previous section, we have discussed the effects of a finite $l_{B}$ in analogy with the temporal size $L_{\tau}$. Here we make a comparison of the magnetic catalysis as a finite-size effect in spatial directions with the conventional finite-size effects. In a finite-size Dirac system with an isotropic linear system size $L$ in absence of a magnetic field, an order parameter $M$ (more precisely, a long-range order $M=\sqrt{\left\langle\hat{M}^{2}\right\rangle}$ ) is usually overestimated when compared with the thermodynamic value, and it shows smooth crossover for a wide range of interaction strength when the system size is fixed [1-13]. For different system sizes, it behaves as $M \sim L^{-\beta / v}$ at the critical point based on the conventional finite-size scaling ansatz. Similar scaling relations hold also for an infinite system within a framework of tensor network states where the system size $L$ is replaced by the correlation length due to a finite bond dimension (see also Appendix B) [14,58]. In this sense, at least formally, the enhanced $M$ by the magnetic field in the present study is analogous to the overestimated $M$ in a conventional finite-size system without a magnetic field. Furthermore, these two phenomena share a physical origin in common, i.e., the dimensional reduction. As mentioned in Sec. I, a magnetic field reduces the spatial dimensionality $d \rightarrow d-2$ via the Landau quantization. Similarly, a small system size quantizes the spatial degrees of freedom and possible wave numbers are discretized. Consequently, the density of states at low energy can become larger than that in the thermodynamic limit and correlation effects can be amplified, which would lead to enhanced or overestimated $M$. Therefore, the magnetic catalysis can be regarded as a finite-size effect and is expected to be a quite universal phenomenon. However, there is a crucial difference that the finite $l_{B}$ effect can be observed in an experiment as an anomalous $B$ dependence $M(B) \sim$ $l_{B}^{-\beta / v} \sim B^{\beta / 2 v}$, in contrast to the familiar finite-size scaling, $M \sim L^{-\beta / \nu}$.

\section{Ground-state energy density}

Although we have been focusing on the CDW order parameter, scaling behaviors will also be seen in other quantities such as the ground-state energy density $\varepsilon$ itself. According to Eq. (3), $\varepsilon$ of a thermodynamically large system is expected to behave as

$$
\varepsilon\left(g, l_{B}^{-1}\right)=\varepsilon(g, 0)+\frac{\varepsilon_{\text {sing }}\left(g l_{B}^{1 / \nu}\right)}{l_{B}^{3}}+\cdots .
$$

At the quantum critical point $g=0$ (i.e., $V=V_{c}$ ), the prefactor in front of $l_{B}^{-3}$ might be factorized as $\varepsilon_{\text {sing }}(0)=C_{0} v$ with a constant $C_{0}$ and the "speed of light" $v$ characterizing the underlying field theory with the Lorentz invariance [65]. Away from the quantum critical point, the mean-field behaviors will be qualitatively correct as we have seen in the CDW order parameter $M$ (Sec. III). Indeed, our iDMRG calculation and mean-field calculation suggest for a small magnetic field $l_{B}^{-1} \rightarrow 0, \varepsilon_{\text {sing }}\left(g l_{B}^{1 / \nu} \ll-1\right) \sim$ const $>0$ in the Dirac semimetal regime $g<0$ (i.e. $\left.V<V_{c}\right)$, while $\varepsilon_{\text {sing }}\left(g l_{B}^{1 / \nu} \gg\right.$ 1) $\sim l_{B}^{-1}>0$ in the ordered phase $g>0$ (i.e., $V>V_{c}$ ), which is in agreement with the large $N_{f}$ field theory [24,25]. Consequently, the orbital magnetic moment $m_{\text {orb }}=-\partial \varepsilon / \partial B$ will be $m_{\text {orb }} \sim-\sqrt{B}$ for the former (and also at the critical point) and $m_{\text {orb }} \sim-B$ for the latter. Details of the ground-state energy 
density and the diamagnetic orbital magnetic moment will be discussed elsewhere.

\section{Finite temperature correction}

Finally, we briefly touch on finite temperature effects around $T=0$. At finite temperature, the new length scale $L_{\tau}$ is introduced, and we expect an anomalous $T / \sqrt{B}$ scaling in our system, by following a scaling hypothesis for the singular part of the free energy density, $f_{\text {sing }}\left(g, h, l_{B}^{-1}, L_{\tau}^{-1}\right)=$ $b^{-D} f_{\text {sing }}\left(b^{y_{g}} g, b^{y_{h}} h, b l_{B}^{-1}, b^{z} L_{\tau}^{-1}\right)$ with $z=1$. For example, the CDW order parameter would have a finite temperature correction given by $M(B, T)=B^{\beta / 2 v} \Psi(T / \sqrt{B})$ at the critical point $g=0$, where $\Psi(\cdot)$ is a scaling function with the property $\Psi(x \rightarrow 0)=$ const. Since finite temperature effects are important in experiments, detailed investigations of them would be an interesting future problem.

\section{SUMMARY}

We have discussed quantum criticality of the magnetic catalysis in spinless fermions on the $\pi$-flux square lattice by nonperturbative calculations with iDMRG. We found the scaling behavior of the CDW order parameter $M(B)$ characteristic of the $(2+1)$-dimensional chiral Ising universality class, and established a global phase diagram near the quantum critical point. The present study is a first nonperturbative investigation of fermionic quantum criticality under a magnetic field and could provide a firm basis for deeper understandings of other related systems.

\section{ACKNOWLEDGMENTS}

We thank F. Pollmann for introducing the open source code TenPy for the iDMRG calculations. We are also grateful to Y. Fuji, M. Oshikawa, and K. Fukushima for valuable discussions. The numerical calculations were performed at Max Planck Institute for the Physics of Complex Systems. This work was supported by JSPS KAKENHI Grant No. JP17J05736, No. JP17K14333, and KAKENHI on Innovative Areas "J-Physics" (No. JP18H04318).

\section{APPENDIX A: QUICK OVERVIEW OF IDMRG}

In this Appendix, we briefly touch on the basics of iDMRG [51-56]. The DMRG is a variational method to accurately simulate a target quantum state based on the framework of matrix product states. A ground state in a one-dimensional system can be expressed in the form of a matrix product state,

$$
|\Psi\rangle=\sum_{i_{1}, \cdots, i_{N}} \operatorname{Tr}\left[M^{[1] i_{1}} \cdots M^{[N] i_{N}}\right]\left|i_{1}, \cdots, i_{N}\right\rangle,
$$

where $N$ is the system size and $\left\{\left|i_{1}, \ldots, i_{N}\right\rangle\right\}$ is a local basis. The matrix $M$ is decomposed to $M=U \Lambda V^{\dagger}$ by the singular value decomposition, and only the largest $\chi$ singular values in the diagonal matirx $\Lambda$ are kept in numerical calculations. This works quite well particularly for a gapped system where the singular values decay exponentially in $\chi$. The optimal matrices are found by minimizing the variational state energy.

In iDMRG, we assume that the matrices $\left\{M^{[k]}\right\}$ have a periodicity $N^{\prime}$, such that $M^{[k]}=M^{\left[k+N^{\prime}\right]}$. This enables us to formally consider an infinitely large system by repeating the unit cell structure,

$$
\begin{aligned}
|\Psi\rangle= & \sum \operatorname{Tr}\left[\cdots M^{\left[N^{\prime}\right] i_{0}} M^{[1] i_{1}} \cdots M^{\left[N^{\prime}\right] i_{N^{\prime}}} M^{[1] i_{N^{\prime}+1}} \cdots\right] \\
& \times\left|\ldots, i_{0}, i_{1}, \ldots, i_{N^{\prime}}, i_{N^{\prime}+1}, \ldots\right\rangle .
\end{aligned}
$$

One can also use this scheme to study a two-dimensional system by introducing a "snake-like trace" of the twodimensional lattice and regarding it as a one-dimensional system with long-range hopping and interactions. In our study, we consider $L_{x} \times L_{y}=\infty \times L_{y}$ system with the period $L_{x}^{\prime}$ along the $x$ direction. This system can be regarded as an infinite one-dimensional system with the period $N^{\prime}=L_{x}^{\prime} \times$ $L_{y}$, and such a one-dimensional system can be described by a matrix product state. Detailed discussions and applications can be found in the literature [51-56].

\section{APPENDIX B: TWO-LEG LADDER}

We briefly discuss numerical results for a two-leg ladder system at half-filling for a comparison. Here we do not use the $\chi \rightarrow \infty$ extrapolation but instead apply correlation length scaling for several values of $\chi$. The two-leg ladder system has been extensively investigated with and without magnetic fields $[58,70]$, but criticality of a magnetic-field-induced order has not been examined before to our best knowledge. We consider the Hamiltonian (1), where, instead of the string gauge, the hopping integrals are now $t_{i j}=t e^{i \phi / 2}$ along the
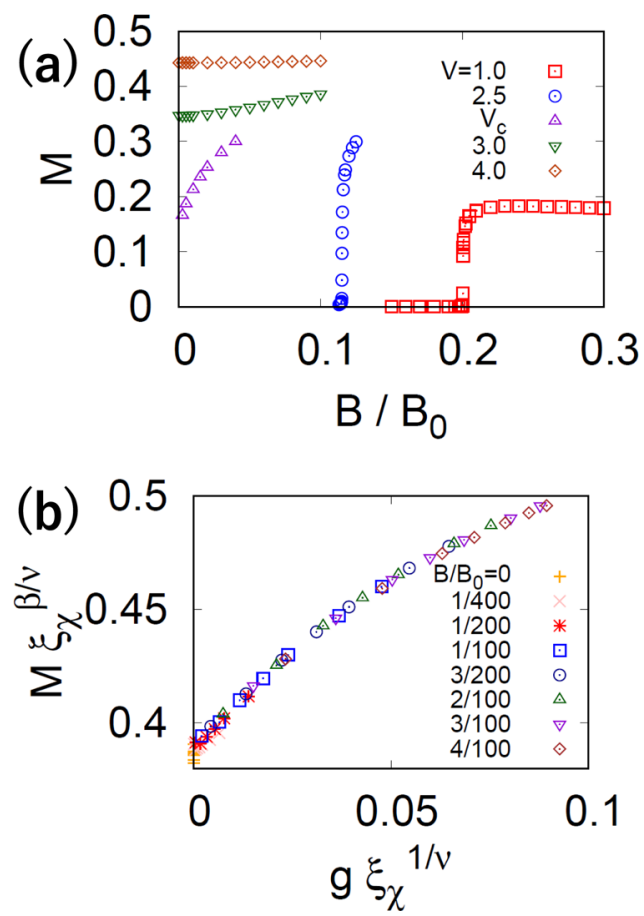

FIG. 6. (a) The CDW order parameter $M$ for several values of the interaction $V$ calculated by iDMRG with the fixed bond dimension $\chi=200$. (b) The scaling plot of $M$ at the critical point $V=V_{c}=$ $2.8678 t$ in terms of the reduced coupling $g=\left(B / B_{0}\right)^{2}$ and the correlation length due to the finite bond dimension $\xi_{\chi}$. The critical exponents are fixed as $\beta=0.125$ and $v=1$. The bond dimensions used in the calculation are $\chi=20-200$. 
chain-1, $t_{i j}=-t e^{-i \phi / 2}$ along the chain-2, and the interchain hopping $t_{i j}=t$. This realizes a magnetic field $B=\phi / a^{2}$ with the lattice constant $a=1$, but note that the magnetic length plays no role in the present two-leg ladder since the system size in the $y$ direction is only $L_{y}=2$.

Figure 6(a) shows the CDW order parameter $M$ as a function of the magnetic field $B$ for various interaction strengths. Differently from the cylinder geometry discussed in the main text, $M$ remains zero for some range of $B \neq$ 0 when the interaction $V$ is smaller than the critical value $V_{c}\left(L_{y}=2\right)=2.8678 t$. The field-induced phase transitions for
$V<V_{c}\left(L_{y}=2\right)$ are so sharp that it is difficult to numerically identify the nature of these phase transitions. On the other hand, $M(B)$ at the critical point exhibits the conventional $(1+$ 1)-dimensional Ising criticality with the critical exponents $\beta=0.125, v=1$ as shown in Fig. 6(b). Here the reduced coupling constant is chosen as $g=\left(B / B_{0}\right)^{2}$, and the cutoff length scale is the correlation length due to the finite bond dimension $\xi_{\chi}$ computed from the second largest eigenvalue of the transfer matrix. The Ising criticality is consistent with the previous study for the two-leg ladder with no magnetic field whose criticality is described by free Marajona fermions [58].
[1] S. Sorella and E. Tosatti, Europhys. Lett. 19, 699 (1992).

[2] F. F. Assaad and I. F. Herbut, Phys. Rev. X 3, 031010 (2013).

[3] L. Wang, P. Corboz, and M. Troyer, New J. Phys. 16, 103008 (2014).

[4] L. Wang, Y.-H. Liu, and M. Troyer, Phys. Rev. B 93, 155117 (2016).

[5] Z.-X. Li, Y.-F. Jiang, and H. Yao, Phys. Rev. B 91, 241117(R) (2015).

[6] Z.-X. Li, Y.-F. Jiang, and H. Yao, New J. Phys. 17, 085003 (2015).

[7] S. Hesselmann and S. Wessel, Phys. Rev. B 93, 155157 (2016).

[8] E. Huffman and S. Chandrasekharan, Phys. Rev. D 96, 114502 (2017).

[9] M. Hohenadler, F. Parisen Toldin, I. F. Herbut, and F. F. Assaad, Phys. Rev. B 90, 085146 (2014).

[10] F. Parisen Toldin, M. Hohenadler, F. F. Assaad, and I. F. Herbut, Phys. Rev. B 91, 165108 (2015).

[11] Y. Otsuka, S. Yunoki, and S. Sorella, Phys. Rev. X 6, 011029 (2016).

[12] Y. Otsuka, K. Seki, S. Sorella, and S. Yunoki, Phys. Rev. B 98, 035126 (2018).

[13] Z. Zhou, C. Wu, and Y. Wang, Phys. Rev. B 97, 195122 (2018).

[14] P. Corboz, P. Czarnik, G. Kapteijns, and L. Tagliacozzo, Phys. Rev. X 8, 031031 (2018).

[15] J. Braun, J. Phys. G: Nucl. Part. Phys. 39, 033001 (2012).

[16] B. Rosenstein, H.-L. Yu, and A. Kovner, Phys. Lett. B 314, 381 (1993).

[17] L. Rosa, P. Vitale, and C. Wetterich, Phys. Rev. Lett. 86, 958 (2001).

[18] I. F. Herbut, Phys. Rev. Lett. 97, 146401 (2006).

[19] I. F. Herbut, V. Juričić, and O. Vafek, Phys. Rev. B 80, 075432 (2009).

[20] L. Janssen and I. F. Herbut, Phys. Rev. B 89, 205403 (2014).

[21] B. Ihrig, L. N. Mihaila, and M. M. Scherer, Phys. Rev. B 98, 125109 (2018).

[22] R. Boyack, H. Yerzhakov, and J. Maciejko, arXiv:2004.09414.

[23] Generally, a long-range interaction affects criticality of a phase transition. However, it was argued that the long-range Coulomb interaction does not affect the criticality of a phase transition driven by a short-range interaction in a Dirac system $[9,19]$.

[24] I. A. Shovkovy, in Strongly Interacting Matter in Magnetic Fields, Lecture Notes in Physics Vol. 871, edited by D. Kharzeev, K. Landsteiner, A. Schmitt, and H. Yee (Springer, New York, 2013).
[25] V. A. Miransky and I. A. Shovkovy, Phys. Rep. 576, 1 (2015).

[26] J. O. Andersen, W. R. Naylor, and A. Tranberg, Rev. Mod. Phys. 88, 025001 (2016).

[27] K. Fukushima, Prog. Part. Nucl. Phys. 107, 167 (2019).

[28] V. P. Gusynin, V. A. Miransky, and I. A. Shovkovy, Phys. Rev. Lett. 73, 3499 (1994).

[29] V. Gusynin, V. Miransky, and I. Shovkovy, Nucl. Phys. B 462, 249 (1996).

[30] K. Fukushima and J. M. Pawlowski, Phys. Rev. D 86, 076013 (2012).

[31] D. D. Scherer and H. Gies, Phys. Rev. B 85, 195417 (2012).

[32] P. V. Buividovich, M. N. Chernodub, E. V. Luschevskaya, and M. I. Polikarpov, Phys. Rev. D 80, 054503 (2009).

[33] M. D’Elia, S. Mukherjee, and F. Sanfilippo, Phys. Rev. D 82, 051501(R) (2010).

[34] E.-M. Ilgenfritz, M. Kalinowski, M. Müller-Preussker, B. Petersson, and A. Schreiber, Phys. Rev. D 85, 114504 (2012).

[35] G. S. Bali, F. Bruckmann, G. Endrődi, Z. Fodor, S. D. Katz, and A. Schäfer, Phys. Rev. D 86, 071502(R) (2012).

[36] D. V. Khveshchenko, Phys. Rev. Lett. 87, 206401 (2001).

[37] E. V. Gorbar, V. P. Gusynin, V. A. Miransky, and I. A. Shovkovy, Phys. Rev. B 66, 045108 (2002).

[38] G. W. Semenoff, I. A. Shovkovy, and L. C. R. Wijewardhana, Mod. Phys. Lett. A 13, 1143 (1998).

[39] I. F. Herbut and B. Roy, Phys. Rev. B 77, 245438 (2008).

[40] B. Roy and I. F. Herbut, Phys. Rev. B 83, 195422 (2011).

[41] B. Roy, M. P. Kennett, and S. Das Sarma, Phys. Rev. B 90, 201409(R) (2014).

[42] D. L. Boyda, V. V. Braguta, S. N. Valgushev, M. I. Polikarpov, and M. V. Ulybyshev, Phys. Rev. B 89, 245404 (2014).

[43] C. DeTar, C. Winterowd, and S. Zafeiropoulos, Phys. Rev. Lett. 117, 266802 (2016).

[44] C. DeTar, C. Winterowd, and S. Zafeiropoulos, Phys. Rev. B 95, 165442 (2017).

[45] M. Hirata, K. Ishikawa, G. Matsuno, A. Kobayashi, K. Miyagawa, M. Tamura, C. Berthier, and K. Kanoda, Science 358, 1403 (2017).

[46] J. Fujioka, R. Yamada, M. Kawamura, S. Sakai, M. Hirayama, R. Arita, T. Okawa, D. Hashizume, M. Hoshino, and Y. Tokura, Nat. Commun. 10, 362 (2019).

[47] C. Sow, S. Yonezawa, S. Kitamura, T. Oka, K. Kuroki, F. Nakamura, and Y. Maeno, Science 358, 1084 (2017). 
[48] Y. Xu, C. Yue, H. Weng, and X. Dai, Phys. Rev. X 7, 011027 (2017).

[49] J. Dalibard, F. Gerbier, G. Juzeliūnas, and P. Öhberg, Rev. Mod. Phys. 83, 1523 (2011).

[50] Although there are extensive nonperturbative lattice Monte Carlo simulations for magnetic catalysis in QCD [32-35] and also in QED-like models for graphene [42-44], their quantum critical properties have not been examined before to our best knowledge.

[51] S. R. White, Phys. Rev. Lett. 69, 2863 (1992).

[52] U. Schollwöck, Rev. Mod. Phys. 77, 259 (2005).

[53] U. Schollwöck, Ann. Phys. 326, 96 (2011).

[54] I. P. McCulloch, arXiv:0804.2509.

[55] J. A. Kjäll, M. P. Zaletel, R. S. K. Mong, J. H. Bardarson, and F. Pollmann, Phys. Rev. B 87, 235106 (2013).

[56] J. Hauschild and F. Pollmann, SciPost Phys. Lect. Notes, 5 (2018).

[57] Y. Hatsugai, K. Ishibashi, and Y. Morita, Phys. Rev. Lett. 83, 2246 (1999).

[58] Y. Tada, Phys. Rev. B 100, 125145 (2019).

[59] In the mean-field calculation, the interaction term is approximated as $n_{i} n_{j} \rightarrow n_{i}\left\langle n_{j}\right\rangle+\left\langle n_{i}\right\rangle n_{j}-\left\langle n_{i}\right\rangle\left\langle n_{j}\right\rangle$.
[60] J. L. Cardy (ed.), Finite-Size Scaling (Elsevier Science, Amsterdam, 1988).

[61] The order parameter anomalous dimension is $\eta=1$ in the large $N_{f}$ limit, and therefore the hyperscaling relation holds within mean-field approximations in the present $D=3$ system. This is consistent with the result that our scaling ansatz with respect to $l_{B}$ holds at the mean-field level.

[62] D. S. Fisher, M. P. A. Fisher, and D. A. Huse, Phys. Rev. B 43, 130 (1991).

[63] I. D. Lawrie, Phys. Rev. Lett. 79, 131 (1997).

[64] Z. Tesanović, Phys. Rev. B 59, 6449 (1999).

[65] M. Rader and A. M. Läuchli, Phys. Rev. X 8, 031030 (2018).

[66] F. Pollmann, S. Mukerjee, A. M. Turner, and J. E. Moore, Phys. Rev. Lett. 102, 255701 (2009).

[67] S. L. Sondhi, S. M. Girvin, J. P. Carini, and D. Shahar, Rev. Mod. Phys. 69, 315 (1997).

[68] T. Moriya and K. Ueda, Adv. Phys. 49, 555 (2000).

[69] H. v. Löhneysen, A. Rosch, M. Vojta, and P. Wölfle, Rev. Mod. Phys. 79, 1015 (2007).

[70] S. T. Carr, B. N. Narozhny, and A. A. Nersesyan, Phys. Rev. B 73, 195114 (2006). 